\title{
CANONICAL VARIETIES OF COMPLETELY REGULAR SEMIGROUPS
}

\author{
MARIO PETRICH
}

(Received 15 April 2002; revised 1 August 2005)

Communicated by D. Easdown

\begin{abstract}
Completely regular semigroups $\mathcal{C R}$ are regarded here as algebras with multiplication and the unary operation of inversion. Their lattice of varieties is denoted by $\mathcal{L}(\mathcal{C R})$. Let $\mathcal{B}$ denote the variety of bands and $\mathcal{L}(\mathcal{B})$ the lattice of its subvarieties. The mapping $\mathcal{V} \rightarrow \mathcal{V} \cap \mathcal{B}$ is a complete homomorphism of $\mathcal{L}(\mathcal{C R})$ onto $\mathcal{L}(\mathcal{B})$. The congruence induced by it has classes that are intervals, say $\mathcal{V} B=\left[\mathcal{V}_{B}, \mathcal{V}^{B}\right]$ for $\mathcal{V} \in \mathcal{L}(\mathcal{C R})$. Here $\mathcal{V}_{B}=\mathcal{V} \cap \mathcal{B}$. We characterize $\mathcal{V}^{B}$ in several ways, the principal one being an inductive way of constructing bases for $\vee$-irreducible band varieties. We term the latter canonical. We perform a similar analysis for the intersection of these varieties with the varieties $B \mathcal{G}, \mathcal{O B G}$ and $\mathcal{B}$.
\end{abstract}

2000 Mathematics subject classification: primary $20 \mathrm{M} 07$.

Keywords and phrases: completely regular semigroup, variety, band, congruence, interval, Polák's theorem, ladder.

\section{Introduction and summary}

Completely regular semigroups are those that are unions of their (pairwise disjoint maximal) subgroups. In the context of varieties, they are considered as algebras with the binary operation of multiplication and the unary operation of inversion. If $S$ is such a semigroup and $a \in S$, then $a^{-1}$ is the inverse of $a$ in the maximal subgroup of $S$ containing $a$. They form the variety $\mathcal{C R}$. We denote by $\mathcal{L}(\mathcal{C R})$ the lattice of all subvarieties of $\mathcal{C R}$.

The lattice $\mathcal{L}(\mathcal{C R})$ has attracted considerable attention. This is in view of the importance of the class of completely regular semigroups (with or without the unary operation) and the amenability of this lattice to various techniques. The principal of these consists of copious decompositions of $\mathcal{L}(\mathcal{C R})$, whose classes promise a

(C) 2007 Australian Mathematical Society 1446-7887/07 $\$$ A2.00+0.00 
description usually in terms of varieties of groups or bands (idempotent semigroups). This approach to the structure of $\mathcal{L}(\mathcal{C R})$ has produced satisfactory results, but is by no means exhausted. For the study of these decompositions, and the construction of new ones, there is plenty of room for further research.

Denote by $\mathcal{B}$ the variety of bands. Our decomposition of $\mathcal{L}(\mathcal{C R})$ is the complete congruence induced by the mapping $\mathcal{V} \rightarrow \mathcal{V} \cap \mathcal{B}$. Its classes are intervals with the lower end $\mathcal{V} \cap \mathcal{B}$. We concentrate on $\vee$-irreducible band varieties since the others are obtained as finite intersections of these, and this property carries over to upper ends of these intervals. We characterize these in two ways and provide a single identity as a basis for their system of identities. This identity is defined inductively starting with two simple identities and their duals. This system of identities represents a variant of the systems of identities for $v$-irreducible varieties of bands constructed by Gerhard and Petrich [2]. A characterization of upper ends of these intervals in terms of Malcev product was devised by Reilly and Zhang [13]. An interesting extension of these ideas to a class of pseudovarieties was treated by Trotter and Weil [15]. We conclude the paper with a similar analysis for intersections of upper ends with the varieties $\mathcal{B G}$, $\mathcal{O B G}$ and $\mathcal{B}$, providing in each case a basis for their identities consisting of a single identity.

We term the upper ends of intervals belonging to $v$-irreducible band varieties, with a few exceptions at the bottom of the lattice, canonical. They play a seminal role in the context of varieties of completely regular semigroups.

\section{Notation and terminology}

We state here the needed symbolism and a few concepts. A complete list of these can be found in the book [7]. We shall refer to the book [7], as well as to its second volume [8], which is in preparation. In the latter case, we shall also state the original sources. Throughout we use set theoretic notation to describe and manipulate classes such as varieties.

For $\rho$ an equivalence relation on a nonempty set $X$ and $x \in X, x \rho$ denotes the $\rho$-class of $x$. In any lattice $L$, for $a, b \in L$ such that $a \leq b$, define the interval $[a, b]$ by $\{c \in L \mid a \leq c \leq b\}$.

Let $S$ be a semigroup. Then $E(S)$ denotes its set of idempotents and $\mathcal{C}(S)$ the lattice of congruences on $S$. For $\rho \in \mathcal{C}(S)$, the kernel and the trace of $\rho$ are given by $\operatorname{ker} \rho=\{a \in S \mid a \rho e$ for some $e \in E(S)\}, \operatorname{tr} \rho=\left.\rho\right|_{E(S)}$, and the left and the right traces of $\rho$ are $\operatorname{ltr} \rho=\operatorname{tr}(\rho \vee \mathcal{L})^{0}$ and $\operatorname{trt} \rho=\operatorname{tr}(\rho \vee \mathcal{R})^{0}$, where the join is taken within equivalence relations on $S$. On $S$, the relations defined by

$$
\lambda K \rho \Longleftrightarrow \operatorname{ker} \lambda=\operatorname{ker} \rho \text { and } \lambda T_{p} \rho \Longleftrightarrow p \operatorname{tr} \lambda=p \operatorname{tr} \rho,
$$


where $p \in\{l, r\}$, are the kernel, left trace and right trace relations, respectively. Their classes are intervals, and we write

$$
\rho P=\left[\rho_{P}, \rho^{P}\right], \quad P \in\left\{K, T_{l}, T_{r}\right\} .
$$

Finally let $S$ be a completely regular semigroup. As $S$ is a union of its (maximal) subgroups, on $S$ we have a unary operation $a \rightarrow a^{-1}$, where $a^{-1}$ is the inverse of $a$ in the maximal subgroup of $S$ containing $a$. Hence for the purpose of studying varieties of completely regular semigroups, they are considered with the binary operation of multiplication and the unary operation of inversion. We write $a^{0}=a a^{-1}\left(=a^{-1} a\right)$ for any element $a$ of $S$.

We denote by $\mathcal{C R}$ the variety of all completely regular semigroups and by $\mathcal{L}(\mathcal{C R})$ the lattice of its subvarieties. Via the usual antiisomorphism of the lattice of fully invariant congruences on a free completely regular semigroup of infinite rank and $\mathcal{L}(\mathcal{C R})$, the relations $K, T_{l}$ and $T_{r}$ defined above transfer to $\mathcal{L}(\mathcal{C R})$ in an obvious way. We use the same notation for these relations on $\mathcal{L}(\mathcal{C R})$ (and their intervals) as for the corresponding ones on semigroups. For these operators, we write for example, $\mathcal{V}_{K}, \mathcal{V}^{K T_{r}}=\left(\mathcal{V}^{K}\right)^{T_{r}}$, and so on. For $\mathcal{V} \in \mathcal{L}(\mathcal{C R}), \mathcal{L}(\mathcal{V})$ denotes the lattice of all subvarieties of $\mathcal{V}$.

If $\mathcal{V} \in \mathcal{L}(\mathcal{C R})$ has a basis $\left\{u_{\alpha}=v_{\alpha}\right\}_{\alpha \in A}$, we write $\mathcal{V}=\left[u_{\alpha}=v_{\alpha}\right]_{\alpha \in A}$, or simply $\mathcal{V}=[u=v]$ if $A$ is a singleton. We shall mention a great number of subvarieties of $\mathcal{L}(\mathcal{C R})$ using for the notation their standard acronyms, for a full name and bases, we refer to the book [7].

Let $X$ be a nonempty set. If $x_{1}, \ldots, x_{n} \in X$, then $w=x_{1} \cdots x_{n}$ is a word over $X$, $h(w)=x_{1}$ is the head of $w, t(w)=x_{n}$ is the tail of $w$, and $c(w)=\left\{x_{1}, \ldots, x_{n}\right\}$ is the content of $w$.

We start by describing the specific construction needed throughout. Let $\Theta$ be the set of all (nonempty) words over the alphabet $\left\{T_{l}, T_{r}\right\}$ of the form $P_{1} \cdots P_{n}$, where $P_{i} \in\left\{T_{l}, T_{r}\right\}$ and $P_{i} \neq P_{i+1}$ for $i=1, \ldots, n-1$ with multiplication

$$
\left(P_{1} \cdots P_{m}\right)\left(Q_{1} \cdots Q_{n}\right)= \begin{cases}P_{1} \cdots P_{m} Q_{1} \cdots Q_{n} & \text { if } P_{m} \neq Q_{1}, \\ P_{1} \cdots P_{m} Q_{2} \cdots Q_{n} & \text { otherwise }\end{cases}
$$

Clearly $\Theta$ is a semigroup. We adjoin the empty word $\emptyset$ to $\Theta$ to form $\Theta^{1}$. We now coordinatize $\Theta$ as follows.

For $p \in\{l, r\}$ and $n \geq 1$, let $\langle p, n\rangle$ be the product of $n$ factors of alternating $T_{l}$ and $T_{r}$ starting with $T_{p}$. We also write $\bar{p}=q$, where $\{p, q\}=\{l, r\}$. For these symbols, we have the product

$$
\langle p, m\rangle\langle q, n\rangle= \begin{cases}\langle p, m+n\rangle & \text { if } p=q, m \text { is even or } p \neq q, m \text { is odd } \\ \langle p, m+n-1\rangle & \text { otherwise }\end{cases}
$$


and the dual

$$
\overline{\langle p, n\rangle}= \begin{cases}\langle p, n\rangle & \text { if } n \text { is odd, } \\ \langle\bar{p}, n\rangle & \text { if } n \text { is even. }\end{cases}
$$

We now return to $\mathcal{L}(\mathcal{C R})$. First let $\mathcal{K}_{0}=\left\{\mathcal{V}_{K} \mid \mathcal{V} \in \mathcal{L}(\mathcal{C R})\right\}$. Then $\mathcal{K}_{0}$ is a complete lattice whose joins coincide with those in $\mathcal{L}(\mathcal{C R})$. We adjoin three elements to $\mathcal{K}_{0}$ by letting $\mathbb{N}_{3}^{*}=\left\{L^{*}, T^{*}, R^{*}\right\}, \mathcal{K}=\mathcal{K}_{0} \cup \mathbb{N}_{3}^{*}$ with the ordering in $\mathcal{K}_{0}$, and for any $\mathcal{V} \in \mathcal{K}_{0}$,

$$
\mathcal{V}>L^{*}>T^{*}<R^{*}<\mathcal{V}
$$

Also let $\mathbb{N}_{3}=\{\mathcal{L N B}, \mathcal{S}, \mathcal{R N} \mathcal{B}\}$ and $\mathbb{N}_{6}=\{\mathcal{L Z}, \mathcal{T}, \mathcal{R Z}\} \cup \mathbb{N}_{3}$.

We may think of the elements $L^{*}, T^{*}$ and $R^{*}$ as 'markers'. In addition, we have the following 'markers' for the operators of $K$ upper and lower. For any $\mathcal{V} \in \mathcal{L}(\mathcal{C R})$, we define

$$
\mathcal{V}_{K^{*}}= \begin{cases}\mathcal{V}_{K} & \text { if } \mathcal{V} \notin \mathbb{N}_{6}, \\ L^{*} & \text { if } \mathcal{V} \in\{\mathcal{L} \mathcal{Z}, \mathcal{L N B}\} \\ T^{*} & \text { if } \mathcal{V} \in\{\mathcal{T}, \mathcal{S}\} \\ R^{*} & \text { if } \mathcal{V} \in\{\mathcal{R Z}, \mathcal{R N} \mathcal{B}\}\end{cases}
$$

and for any $\mathcal{V} \in \mathcal{K}$,

$$
\mathcal{V}^{K^{*}}= \begin{cases}\mathcal{V}^{K} & \text { if } \mathcal{V} \in \mathcal{K}_{0} \\ \mathcal{L} \mathcal{N} \mathcal{B} & \text { if } \mathcal{V}=L^{*} \\ \mathcal{S} & \text { if } \mathcal{V}=T^{*} \\ \mathcal{R N \mathcal { B }} & \text { if } \mathcal{V}=R^{*}\end{cases}
$$

In particular, for any $\mathcal{V} \in \mathcal{L}(\mathcal{C R})$ and $\tau \in \Theta$, we write

$$
\mathcal{V}_{\tau K^{*}}=\left(\mathcal{V}_{\tau}\right)_{K^{*}} \text { and } \mathcal{V}^{K^{*} \tau}=\left(\mathcal{V}^{K^{*}}\right)^{\tau}
$$

The set $\mathcal{K}^{\Theta^{1}}$ of all functions $\Theta^{1} \rightarrow \mathcal{K}$, ordered componentwise, is a complete lattice. Recall that both $\Theta^{1}$ and $\mathcal{K}$ are partially ordered sets. We now identify a part of $\mathcal{K}^{\boldsymbol{\Theta}^{1}}$.

Let $\Phi$ be the set of all functions $\varphi: \Theta^{1} \rightarrow \mathcal{K}$ satisfying the following conditions:

(P1) $\emptyset \varphi \in \mathcal{K}_{0}$.

(P2) $\varphi$ is order preserving.

(P3) If $\tau \in \Theta$ and $\tau \varphi=L^{*}$, then $t(\tau)=T_{r}$.

(P4) If $\tau \in \Theta$ and $\tau \varphi=R^{*}$, then $t(\tau)=T_{i}$.

(P5) If $\sigma \in \Theta^{1}$ and $\tau \in \Theta$ are such that $\sigma \varphi \in \mathcal{K}_{0}$ and either $\sigma=\emptyset$ or $t(\sigma) \neq h(\tau)$, then $(\sigma \varphi)_{\tau K^{*}} \subseteq(\sigma \tau) \varphi$.

We retain the set theoretical symbolism for $\mathcal{K}$. 


\section{Preparation}

We now state the results most frequently used throughout the paper; for infrequent results, we refer to the books $[7,8]$ or the original sources. The central theorem is due to Polák [10] which we state in the following form.

THEOREM 3.1. The mappings $\chi: \mathcal{V} \rightarrow \chi_{\mathcal{V}}$, where $\chi_{\mathcal{V}}: \tau \rightarrow \mathcal{V}_{\tau K^{*}}(\tau \in \Theta)$ and $\emptyset \rightarrow \mathcal{V}_{K}$, and $\xi: \varphi \rightarrow \bigcap_{\tau \in \Theta^{1}}(\tau \varphi)^{K^{*} \bar{i}}$ are mutually inverse isomorphisms between $[\mathcal{S}, \mathcal{C R}]$ and $\Phi$.

PROOF. See [8] or the original work [10].

It is convenient to think of $\chi_{\mathcal{V}}$ as a ladder in the form

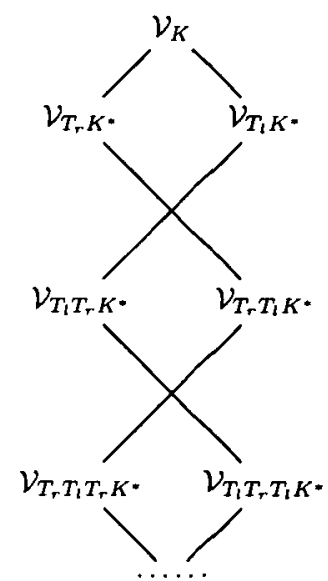

We start with statements concerning $K$.

LEMMA 3.2 .

(i) The following intervals are $K$-classes: $[\mathcal{T}, \mathcal{B}],[\mathcal{G}, \mathcal{O}],[\mathcal{C S}, L \mathcal{O}]$.

(ii) For any $\mathcal{V} \in \mathcal{L}(\mathcal{C R})$,

$$
\mathcal{V}_{K}= \begin{cases}\mathcal{V} \cap \mathcal{G} & \text { if } \mathcal{V} \subseteq \mathcal{O} \\ \mathcal{V} \cap \mathcal{C S} & \text { if } \mathcal{V} \in \mathcal{L}(L \mathcal{O}) \backslash \mathcal{L}(\mathcal{O})\end{cases}
$$

(iii) For any $\mathcal{U}, \mathcal{V} \in \mathcal{L}(\mathcal{C R})$ such that $\mathcal{U} \subseteq \mathcal{V}$, we have $\mathcal{U}_{K} \subseteq \mathcal{V}_{K}$ and $\mathcal{U}^{K} \subseteq \mathcal{V}^{K}$.

PROOF. These statements follow from [8], for their origin, see $[4,6,9,11]$.

We now consider $T_{r}$. The statements concerning $T_{l}$ are obtained by (left-right) duality. 
LEMMA 3.3. (i) The following intervals are $T_{r}$-classes:

$$
\begin{aligned}
& \text { [T, } \mathcal{T} \mathcal{G}], \\
& \mathcal{R G}=\left[a=x^{0} a\right], \\
& {[\mathcal{L Z}, \mathcal{C S}] \text {, }} \\
& \mathcal{C S}=\left[a=(a x)^{0} a\right], \\
& {[\mathcal{S}, \mathcal{R R O} \text { ], }} \\
& \mathcal{R} \mathcal{R O}=\left[x a=a^{0} x a\right], \\
& {[\mathcal{L N B}, L \mathcal{R R O} \text {, }} \\
& L \mathcal{R R O}=\left[y x a=(y a)^{0} y x a\right] .
\end{aligned}
$$

(ii) If $\mathcal{V}=\left[u_{\alpha}=v_{\alpha}\right]_{\alpha \in A} \in \mathcal{L}(\mathcal{C R})$, then $\mathcal{V}^{T_{r}}=\left[u_{\alpha} x=\left(v_{\alpha} x\right)^{0} u_{\alpha} x\right]_{\alpha \in A}$, where $x \notin \bigcup_{\alpha \in A} c\left(u_{\alpha} v_{\alpha}\right)$.

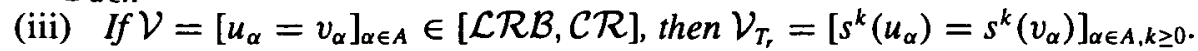

(iv) For any $\mathcal{U}, \mathcal{V} \in \mathcal{L}(\mathcal{C R})$ such that $\mathcal{U} \subseteq \mathcal{V}$, we have $\mathcal{U}_{T_{r}} \subseteq \mathcal{V}_{T_{r}}$ and $\mathcal{U}^{T_{r}} \subseteq \mathcal{V}^{T_{r}}$.

Proof. These statements follow from [8], for their origin, see Petrich and Reilly [7, Theorem IX.5.1], [4], [6] and [10].

Figure 1 depicts the lattice of proper band varieties as constructed in [2].

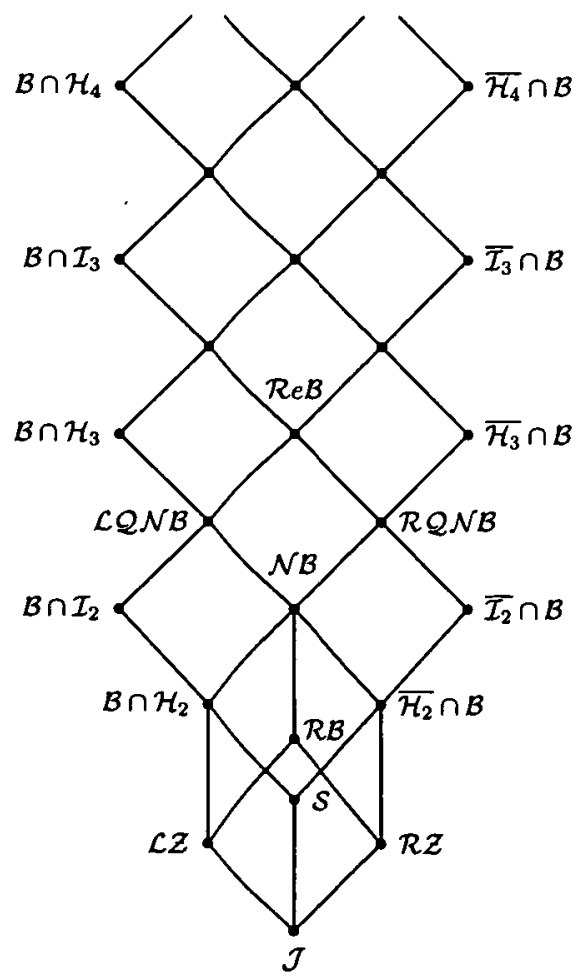

FIGURE 1. Proper band varieties 


\section{Canonical varieties}

We define the canonical varieties by means of their bases. We characterize some of their $T_{l}$ - and $T_{r}$-classes and establish their relationship with the varieties in $\mathbb{N}_{3}$, as well as their ordering.

We start with two operations on the free unary semigroups $U_{X}$ on $X$ by stipulating

$$
\begin{array}{ll}
\bar{x}=x, \quad \overline{u v}=\bar{u} \bar{v}, \overline{u^{-1}}=\bar{u}^{-1} & \left(x \in X, u, v \in U_{X}\right), \\
w^{0}=w w^{-1} & \left(w \in U_{X}\right) .
\end{array}
$$

It follows that $\overline{w^{0}}=\bar{w}^{0}$. The effect of the bar operation is to reverse the order of variables in $w$, while respecting the parentheses and exponents.

We introduce three systems of words $G_{n}, I_{n}$ and $H_{n}$ for $n \geq 2$ inductively as follows:

$$
G_{2}=x_{2} x_{1}, \quad I_{2}=x_{2} x_{1} x_{2}^{0}, \quad H_{2}=x_{2},
$$

and for $n>2$,

$$
G_{n}=x_{n} \overline{G_{n-1}}, \quad P_{n}=G_{n}\left(x_{n} \overline{P_{n-1}}\right)^{0} \quad(P \in\{I, H\}) .
$$

We call the varieties

$$
\begin{aligned}
\mathcal{I}_{n} & =\left[G_{n}=I_{n}\right], & \overline{\mathcal{I}_{n}}=\left[\overline{G_{n}}=\overline{I_{n}}\right], & n \geq 2, \\
\mathcal{H}_{n} & =\left[G_{n}=H_{n}\right], & \overline{\mathcal{H}_{n}}=\left[\overline{G_{n}}=\overline{H_{n}}\right], & n \geq 3, \\
\mathcal{H}_{2} & =\mathcal{L} \mathcal{N O}, & \overline{\mathcal{H}_{2}}=\mathcal{R} \mathcal{N O} &
\end{aligned}
$$

canonical, for they will play an essential role in much of the discussion that follows.

When using canonical varieties, we may consider the alphabet $X=\left\{x_{1}, x_{2}, \ldots\right\}$, or include further variables if it is convenient to change the notation.

We shall frequently have (left-right) duals of statements or formulae involving terms with bars. These are obtained by putting a bar on $\mathcal{P}_{n}, \overline{\mathcal{P}_{n}}, G_{n}, \overline{G_{n}}, P_{n}$ or $\overline{P_{n}}$ for $P \in\{I, H\}$ where $\overline{\bar{W}}=W$.

In the next proposition we calculate $T_{l}$-and $T_{r}$-classes of canonical varieties. When restricted to the lattice of proper band varieties, this result coincides with Gerhard and Petrich [1, Corollary 7.10] and exhausts all $T_{l^{-}}$and $T_{r}$-classes of proper band varieties within $\mathcal{L}(\mathcal{B})$.

PROPOSITION 4.1. Let $\mathcal{P}_{n}=\mathcal{I}_{n}$ for $n \geq 2$ and $\mathcal{P}_{n}=\mathcal{H}_{n}$ for $n>2$.

(i) The following intervals are $T_{1}$-classes: $\left[\mathcal{S}, \mathcal{I}_{2}\right],\left[\mathcal{R N B}, \mathcal{H}_{3}\right],\left[\mathcal{P}_{n}, \mathcal{P}_{n+1}\right]$.

(ii) The following intervals are $T_{r}$-classes: $\left[\mathcal{S}, \overline{\mathcal{I}_{2}}\right],\left[\mathcal{L N} \mathcal{B}, \overline{\mathcal{H}_{3}}\right],\left[\mathcal{P}_{n}, \overline{\mathcal{P}_{n+1}}\right]$. 
Proof. By duality, it suffices to prove part (ii). That $\left[\mathcal{S}, \overline{\mathcal{I}_{2}}\right]$ is a $T_{r}$-class is part of Lemma 3.3 (i). By Petrich and Reilly [7, Theorem IX.5.1 (iv)], we have $L \mathcal{R R O}=\overline{\mathcal{H}_{3}}$ and Lemma 3.3 (i) implies that $\left[\mathcal{L N B}, \overline{\mathcal{H}_{3}}\right]$ is a $T_{r}$-class. We verify first the lower end $\mathcal{P}_{n}$. To apply Lemma 3.3 (iii), we must check that $\mathcal{L R B} \subseteq \mathcal{P}_{n}$. By Petrich and Reilly [7, Lemma V.1.4] we have that $\mathcal{L R B}$ is generated by $L_{2}^{1}$, the 2-element left zero semigroup with an identity adjoined. For this, it suffices to show that $L_{2}^{1}$ satisfies the identity $G_{n}=P_{n}$. The form of this identity is $x_{2} x_{1}=x_{2} x_{1} x_{2}^{0}$ for $\mathcal{P}_{2}=\mathcal{I}_{2}$ and $G_{n}=G_{n}\left(x_{n} \bar{P}_{n-1}\right)^{0}$ otherwise. Hence these identities are of the form $G_{n}=G_{n} W$ for a word $W$ with $c(W) \subseteq c\left(G_{n}\right)$. It is now obvious that $L_{2}^{1}$ satisfies $G_{n}=P_{n}$. It follows by Lemma 3.3 (iii) that $\left(\mathcal{P}_{n}\right)_{T_{r}}=\mathcal{P}_{n}$. For the upper end, by Lemma 3.3 (ii) we get

$$
\begin{aligned}
\mathcal{P}_{n}^{T_{r}} & =\left[G_{n} x_{n+1}=\left(P_{n} x_{n+1}\right)^{0} G_{n} x_{n+1}\right] \\
& =\left[\overline{x_{n+1} \overline{G_{n}}}=\overline{x_{n+1} \overline{G_{n}}\left(x_{n+1} \overline{P_{n}}\right)^{0}}\right]=\left[\overline{G_{n+1}}=\overline{P_{n+1}}\right]=\overline{\mathcal{P}_{n+1}},
\end{aligned}
$$

which proves that $\left[\mathcal{P}_{n}, \overline{\mathcal{P}_{n+1}}\right]$ is a $T_{r}$-class.

It is easy to check that

$\left[\mathcal{S}, \mathcal{I}_{2}\right]$ is the $T_{l}$-class of $\mathcal{H}_{2}, \quad\left[\mathcal{R N B}, \mathcal{H}_{3}\right]$ is the $T_{l}$-class of $\overline{\mathcal{H}_{2}}$, $\left[\mathcal{S}, \overline{\mathcal{I}_{2}}\right]$ is the $T_{r}$-class of $\overline{\mathcal{H}_{2}}, \quad\left[\mathcal{L N B}, \overline{\mathcal{H}_{3}}\right]$ is the $T_{r}$-class of $\mathcal{H}_{2}$.

Hence Proposition 4.1 takes care of all $T_{l}$ - and $T_{r}$-classes of canonical varieties.

The next lemma characterizes $\mathcal{S}^{\tau}, \mathcal{L} \mathcal{N} \mathcal{B}^{\tau}$ and $\mathcal{R N \mathcal { B } ^ { \tau }}$ in terms of canonical varieties. By turning things around, the lemma could be used to define canonical varieties in terms of $\mathcal{S}^{\tau}, \mathcal{L N \mathcal { B } ^ { \tau }}$ and $\mathcal{R N} \mathcal{B}^{\tau}$.

LEMMA 4.2. Let $n \geq 1$.

(i) $\mathcal{S}^{(l, n)}=\mathcal{L} \mathcal{N} \mathcal{B}^{(l, n)}= \begin{cases}\mathcal{I}_{n+1} d & \text { if } n \text { is odd, } \\ \overline{\mathcal{I}_{n+1}} & \text { if } n \text { is even. }\end{cases}$

(ii) $\mathcal{S}^{(r, n)}=\mathcal{R} \mathcal{N B}^{(r, n)}= \begin{cases}\overline{\mathcal{I}_{n+1}} d & \text { if } n \text { is odd, } \\ \mathcal{I}_{n+1} & \text { if } n \text { is even. }\end{cases}$

(iii) $\mathcal{R N B}^{(l, n)}= \begin{cases}\mathcal{H}_{n+2} & \text { if } n \text { is odd, } \\ \mathcal{H}_{n+2} & \text { if } n \text { is even. }\end{cases}$

(iv) $\mathcal{L N B}^{(r, n)}= \begin{cases}\overline{\mathcal{H}_{n+2}} & \text { if } n \text { is odd } \\ \mathcal{H}_{n+2} & \text { if } n \text { is even }\end{cases}$

PROOF. The proof is by induction on $n$.

(i) For $n=1$, by the dual of Lemma 3.3 (i), we have $\mathcal{S}^{T_{I}}=\mathcal{I}_{2}$. Assume the statement is true for $n$. If $n$ is odd, then by Proposition 4.1 (ii), we get

$$
\mathcal{S}^{(l, n+1)}=\left(\mathcal{S}^{(l, n)}\right)^{T_{r}}=\mathcal{I}_{n+1}^{T_{r}}=\overline{\mathcal{I}_{n+2}}
$$


and if $n$ is even, then by Proposition 4.1 (i), we have

$$
\mathcal{S}^{(l, n+1)}=\left(\mathcal{S}^{(l, n)}\right)^{T_{l}}={\overline{\mathcal{I}_{n+1}}}_{T_{l}}=\mathcal{I}_{n+2} \text {. }
$$

This establishes the assertion for $\mathcal{S}^{(l, n)}$. Since $\mathcal{L} \mathcal{N B}^{T_{l}}=\mathcal{S}^{T_{l}}$, the remaining equality also holds.

(ii) This is the dual of part (i).

(iii) The argument here follows the same lines as in the proof of part (i) and is omitted.

(iv) This is the dual of part (iii).

We determine next the ordering of canonical varieties.

LEMMA 4.3. Let $n \geq 2$.

(i) For $\mathcal{P} \in\{\mathcal{H}, \mathcal{I}\}$, we have $\mathcal{P}_{n} \subseteq \overline{\mathcal{P}_{n+1}}$ and $\overline{\mathcal{P}_{n}} \subseteq \mathcal{P}_{n+1}$.

(ii) We have $\mathcal{I}_{n} \subseteq \mathcal{H}_{n+1}, \overline{\mathcal{I}_{n}} \subseteq \overline{\mathcal{H}_{n+1}}, \mathcal{H}_{n} \subseteq \mathcal{I}_{n}$ and $\overline{\mathcal{H}_{n}} \subseteq \overline{\mathcal{I}_{n}}$.

PROOF. (i) This follows directly from Proposition 4.1.

(ii) The proof is by induction on $n$. Since $\mathcal{S} \subset \mathcal{R N} \mathcal{B}$, by the dual of Lemma 3.3 (iv), we have $\mathcal{S}^{T_{l}} \subseteq \mathcal{R} \mathcal{N} \mathcal{B}^{T_{l}}$, which by Lemma 4.2 implies that $\mathcal{I}_{2} \subseteq \mathcal{H}_{3}$. Dually, we get that $\overline{\mathcal{I}_{2}} \subseteq \overline{\mathcal{H}_{3}}$. This establishes the statements for $n=2$. Suppose the validity of both inclusions for $n$. Then by Lemma 3.3 (iv) and its dual, we get $\mathcal{I}_{n}^{T_{r}} \subseteq \mathcal{H}_{n+1}^{T_{r}}$ and $\overline{\mathcal{I}}_{n}{ }^{T} \subseteq \overline{\mathcal{H}}_{n+1} T_{l}$, which by Lemma 4.2 yields $\overline{\mathcal{I}_{n+1}} \subseteq \overline{\mathcal{H}_{n+2}}$, as required.

The argument for the remaining formulae is similar by noting that $\mathcal{H}_{2} \subset \mathcal{I}_{2}$ and starting with $\mathcal{R N B} \subset \mathcal{R R O}$ instead of $\mathcal{S} \subset \mathcal{R N B}$.

The inclusions in Lemma 4.3 are in concordance with implications in Gerhard and Petrich [2, Lemma 6.2].

For some varieties $\mathcal{V}$ of completely regular semigroups, we compare here the varieties $\mathcal{V}_{\langle p, n)}$ with certain varieties in $\mathbb{N}_{6}$. The first lemma concerns varieties of completely simple semigroups.

LEMMA 4.4. Let $\mathcal{V} \in \mathcal{L}(\mathcal{C S})$.

(i) $\mathcal{V}_{(l, n)}= \begin{cases}\mathcal{R Z} & \text { if } n=1 \text { and } \mathcal{V} \supseteq \mathcal{R Z}, \\ \mathcal{T} & \text { otherwise }\end{cases}$

(ii) $\mathcal{V}_{\langle r, n\rangle}= \begin{cases}\mathcal{L Z} & \text { if } n=1 \text { and } \mathcal{V} \supseteq \mathcal{L Z}, \\ \mathcal{T} & \text { otherwise. }\end{cases}$

PROOF. According to Lemma 3.3 (i) and its dual, we have

$$
\mathcal{V}_{T_{l}}=\left\{\begin{array}{ll}
\mathcal{T} & \text { if } \mathcal{V} \subseteq \mathcal{L G}, \\
\mathcal{R Z} & \text { if } \mathcal{V} \supseteq \mathcal{R Z Z},
\end{array} \quad \mathcal{V}_{T_{r}}= \begin{cases}\mathcal{T} & \text { if } \mathcal{V} \subseteq \mathcal{R \mathcal { G }} \\
\mathcal{L Z} & \text { if } \mathcal{V} \supseteq \mathcal{L} \mathcal{Z}\end{cases}\right.
$$


We consider two cases.

(1) $\mathcal{V} \subseteq \mathcal{L G}$. Then $\mathcal{V}_{T_{l}}=\mathcal{T}$ and

$$
\mathcal{V}_{T_{r}}= \begin{cases}\mathcal{T} & \text { if } \mathcal{V} \subseteq \mathcal{G} \\ \mathcal{L} \mathcal{Z} & \text { if } \mathcal{V} \supseteq \mathcal{L Z}\end{cases}
$$

and thus $\mathcal{V}_{T_{r} T_{i}}=\mathcal{T}$.

(2) $\mathcal{V} \supseteq \mathcal{R Z}$. Then $\mathcal{V}_{T_{i}}=\mathcal{R} \mathcal{Z}$, whence $\mathcal{V}_{T_{i} T_{r}}=\mathcal{T}$, and

$$
\mathcal{V}_{T_{r}}= \begin{cases}\mathcal{T} & \text { if } \mathcal{V} \subseteq \mathcal{R G}, \\ \mathcal{L Z} & \text { if } \mathcal{V} \supseteq \mathcal{L} \mathcal{Z},\end{cases}
$$

whence $\mathcal{V}_{T_{i} T_{r}}=\mathcal{T}$.

The assertion of the lemma follows.

The next three lemmas concern arbitrary varieties of completely regular semigroups.

LEMMA 4.5. Let $\mathcal{V} \in \mathcal{L}(\mathcal{C R})$ and $n \geq 1$.

(i) $\mathcal{V}_{(l, n)}=\mathcal{S}$ if and only if $\mathcal{S} \subseteq \mathcal{V} \subseteq \mathcal{I}_{n+1}$.

(ii) $\mathcal{V}_{(r, n)}=\mathcal{S}$ if and only if $\mathcal{S} \subseteq \mathcal{V} \subseteq \overline{\mathcal{I}_{n+1}}$.

PROOF. The argument is by induction on $n$ for both statements simultaneously. For $n=1$, by Lemma 3.3 (i) and its dual, we have

$$
\mathcal{V}_{\tau_{l}}=\mathcal{S} \Longleftrightarrow \mathcal{S} \subseteq \mathcal{V} \subseteq \mathcal{I}_{2}, \quad \mathcal{V}_{T_{r}}=\mathcal{S} \Longleftrightarrow \mathcal{S} \subseteq \mathcal{V} \subseteq \overline{\mathcal{I}_{2}}
$$

Assume that the above statements are true for $n$. Then

$$
\begin{aligned}
\mathcal{V}_{l(, n+1)}=\mathcal{S} & \Longleftrightarrow\left(\mathcal{V}_{T_{i}}\right)_{\langle r, n)}=\mathcal{S} & & \\
& \Longleftrightarrow \mathcal{S} \subseteq \mathcal{V}_{T_{l}} \subseteq \overline{\mathcal{I}_{n+1}} & & \text { by the induction hypothesis } \\
& \Longrightarrow \mathcal{S}^{T_{l}} \subseteq\left(\mathcal{V}_{T_{i}}\right)^{T_{i}} \subseteq\left(\overline{\mathcal{I}_{n+1}}\right)^{T_{l}} & & \text { by Lemma 3.3 (iv) } \\
& \Longrightarrow \mathcal{I}_{2} \subseteq \mathcal{V}^{T_{I}} \subseteq \mathcal{I}_{n+2} & & \text { by Proposition 4.1 (i) } \\
& \Longrightarrow \mathcal{V} \subseteq \mathcal{V}^{T_{I}} \subseteq \mathcal{I}_{n+2} & &
\end{aligned}
$$

and also $\mathcal{S}=\mathcal{V}_{(l, n+1)} \subseteq \mathcal{V}$, so that $\mathcal{S} \subseteq \mathcal{V} \subseteq \mathcal{I}_{n+2}$. Conversely,

$$
\begin{aligned}
& \mathcal{S} \subseteq \mathcal{V} \subseteq \mathcal{I}_{n+2} \Longrightarrow \mathcal{S}_{T_{i}} \subseteq \mathcal{V}_{T_{i}} \subseteq\left(\mathcal{I}_{n+2}\right)_{T_{i}} \text { by Lemma } 3.3 \text { (iv) } \\
& \Longrightarrow \mathcal{S} \subseteq \mathcal{V}_{T_{l}} \subseteq \overline{\mathcal{I}_{n+1}} \quad \text { by Proposition } 4.1 \text { (i) } \\
& \Longrightarrow\left(\mathcal{V}_{T_{l}}\right)_{(r, n)}=\mathcal{S} \quad \text { by the induction hypothesis } \\
& \Longrightarrow \mathcal{V}_{\langle l, n+1\rangle}=\mathcal{S} \text {. }
\end{aligned}
$$

This proves the statement for $\mathcal{V}_{(l, n)}$; a dual argument is valid for $\mathcal{V}_{\langle r, n)}$. 
LEMMA 4.6. Let $\mathcal{V} \in \mathcal{L}(\mathcal{C S})$ and $n \geq 1$.

(i) $\mathcal{V}_{\langle l, n\rangle} \subseteq \mathcal{S}$ if and only if $\mathcal{V} \subseteq \mathcal{I}_{n+1}$.

(ii) $\mathcal{V}_{\langle r, n\rangle} \subseteq \mathcal{S}$ implies $\mathcal{V} \subseteq \overline{\mathcal{I}_{n+1}}$

PROOF. (i) By Lemmas 4.4 and 4.5 , we obtain

$$
\begin{aligned}
\mathcal{V}_{(l, n)} \subseteq \mathcal{S} & \Longleftrightarrow \mathcal{V}_{l l, n)}=\mathcal{S} \text { or } \mathcal{V}_{(l, n)}=\mathcal{T} \\
& \Longleftrightarrow \mathcal{S} \subseteq \mathcal{V} \subseteq \mathcal{I}_{n+1}, \text { or } \mathcal{V} \subseteq \mathcal{L G} \text { and } n=1, \text { or } \mathcal{V} \subseteq \mathcal{C S} \text { and } n>1 \\
& \Longleftrightarrow \mathcal{V} \subseteq \mathcal{I}_{n+1},
\end{aligned}
$$

since $\mathcal{L G} \subseteq \mathcal{I}_{2}$ and $\mathcal{C S} \subseteq \mathcal{I}_{n+1}$ for $n>1$.

(ii) This is the dual of part (i).

LEMMA 4.7. Let $\mathcal{V} \in \mathcal{L}(\mathcal{C R})$ and $\tau \in \Theta$. Then $\mathcal{V}_{\tau K^{*}}=T^{*}$ if and only if $\mathcal{V} \subseteq \mathcal{S}^{\bar{\tau}}$.

PROOF. By Lemma 4.2 (i)-(ii) and Lemma 4.6, we get

$$
\begin{aligned}
\mathcal{V}_{(l, n) K^{*}}=T^{*} & \Longleftrightarrow \mathcal{V}_{\langle l, n)} \subseteq \mathcal{S} \Longleftrightarrow \mathcal{V} \subseteq \mathcal{I}_{n+1} \\
& \Longleftrightarrow \begin{cases}\mathcal{V} \subseteq \mathcal{S}^{(l, n)} & \text { if } n \text { is odd } \\
\mathcal{V} \subseteq \mathcal{S}^{(r, n)} & \text { if } n \text { is even }\end{cases} \\
& \Longleftrightarrow \mathcal{V} \subseteq \mathcal{S}^{(l, n)}
\end{aligned}
$$

and dually for $\mathcal{V}_{\langle r, n\rangle K^{*}}$

\section{The variety $\mathcal{V}^{B}$}

The mapping $\mathcal{V} \rightarrow \mathcal{V} \cap \mathcal{B}$ is a complete retraction of $\mathcal{L}(\mathcal{C R})$ onto $\mathcal{L}(\mathcal{B})$. The classes of the congruence induced on $\mathcal{L}(\mathcal{C R})$ by it are intervals of the form $\left[\mathcal{V} \cap \mathcal{B},(\mathcal{V} \cap \mathcal{B})^{B}\right]$. The main result of this section is the determination of the variety $(\mathcal{V} \cap \mathcal{B})^{B}$.

We start with two lemmas and a figure depicting the ladder of a canonical variety. By Lemma 3.2 (i), we have $\mathcal{T}^{K}=\mathcal{B}$ and by Lemma 3.3 (iii) and its dual, $\mathcal{B}_{T_{i}}=\mathcal{B}_{T_{r}}=\mathcal{B}$. It follows that all the vertices of the ladder of $\mathcal{B}$ are labelled by $\mathcal{T}$. Let $\mathcal{V} \in[\mathcal{S}, \mathcal{C R}]$. If a vertex of the ladder of $\mathcal{V}$ is labelled by an element of $\mathcal{K}_{0}$, then the corresponding vertex of the ladder of $\mathcal{V} \cap \mathcal{B}$ is labelled by $\mathcal{T}$. The remaining vertices of the ladders of $\mathcal{V}$ and $\mathcal{V} \cap \mathcal{B}$ are the same (and are labelled by elements of $\mathbb{N}_{3}^{*}$ ).

LEMMA 5.1. Let $n \geq 2$.

(i) $\left(\mathcal{I}_{n}\right)_{(l, n-1) K^{*}}=\left(\overline{\mathcal{I}_{n}}\right)_{(r, n-1) K^{*}}=T^{*}$.

(ii) $\left(\mathcal{I}_{n}\right)_{(r, n) K^{*}}=\left(\overline{\mathcal{I}_{n}}\right)_{\langle l, n\rangle K^{*}}=T^{*}$. 
PROOF. (i) The proof is by induction on $n$. For $n=2$, we have by Lemma 3.3 (i) that $\left(\mathcal{I}_{2}\right)_{T_{1} K^{*}}=\mathcal{S}_{K^{*}}=T^{*}$ and dually for the second statement. Assume the statements are true for $n$. By Proposition 4.1 (i), we get $\left(\mathcal{I}_{n+1}\right)_{(l, n) K^{*}}=\left(\overline{\mathcal{I}_{n}}\right)_{T_{l}(r, n-1) K^{*}}=T^{*}$ by the induction hypothesis. The statement $\left(\overline{\mathcal{I}_{n+1}}\right)_{(r, n) K^{*}}=T^{*}$ follows dually.

(ii) For $n=2$, we have by Proposition 4.1 (ii) and Lemma 3.3 (i),

$$
\left(\mathcal{I}_{2}\right)_{T_{r} T_{K} K^{*}}=\left(\mathcal{I}_{2}\right)_{T_{i} K^{*}}=\mathcal{S}_{K^{*}}=T^{*}
$$

and for $n>2$, by part (i) and Proposition 4.1 (ii), we have that

$$
\left(\mathcal{I}_{n}\right)_{(r, n) K^{*}}=\left(\left(\mathcal{I}_{n}\right)_{T_{1}}\right)_{(l, n-1) K^{*}}=\left(\mathcal{I}_{n}\right)_{(l, n-1) K^{*}}=T^{*}
$$

and dually for the second statement.

The following lemma is crucial for the main result of the paper.

LEMMA 5.2. Let $\mathcal{U}$ be a canonical variety and $\mathcal{V} \in[\mathcal{S}, \mathcal{C R}]$ be such that $\mathcal{V} \cap \mathcal{B} \subseteq \mathcal{U}$. Then $\mathcal{V} \subseteq \mathcal{U}$.

PROOF. For the four choices for $\mathcal{U}$, namely $\mathcal{I}_{n}, \overline{\mathcal{I}_{n}}, \mathcal{H}_{n}$ and $\overline{\mathcal{H}_{n}}$, we consider only $\mathcal{I}_{n}$ when $n$ is even; the other cases are treated by the same method, but with different details. "We let $\varphi=\chi_{I_{n}}$ with $n$ even and $\psi=\chi \chi_{\mathcal{V}}$. The hypothesis implies that for any $\tau \in \Theta, \tau \varphi \in \mathbb{N}_{3}^{*}$ implies that $\tau \psi=\tau \varphi$. In view of Theorem 3.1, it suffices to prove that $\tau \psi \subseteq \tau \varphi$ whenever $\tau \varphi \in \mathcal{K}_{0}$.

The proof utilizes condition (P5) to obtain bounds on the values of $\psi$, which turns out to be just the corresponding values of $\varphi$. Recall that (P5) is of the form

(i) $\tau \in \Theta$ implies $(\emptyset \psi)_{\tau K^{*}} \subseteq \tau \psi$,

(ii) $\sigma \tau \in \Theta, \sigma \psi \in \mathcal{K}_{0}, t(\sigma) \neq h(\tau)$ implies $(\sigma \psi)_{\tau} K^{*} \subseteq(\sigma \tau) \psi$.

According to the above, we need only consider the case $\sigma \psi \in \mathcal{K}_{0}$. By Lemma 5.1, we have $\langle l, n-1\rangle \varphi=T^{*}$ and thus $\langle l, n-1\rangle \psi=T^{*}$, which by condition (i) yields

$$
(\emptyset \psi)_{(l, n-1) k} \subseteq\langle l, n-1\rangle \psi=T^{*} .
$$

We shall use condition (ii) in the cases: $\sigma \tau=\langle l, n-1)$ and $\sigma \tau=\langle r, n\rangle$. According to Lemma 5.1, we have $(\sigma \tau) \varphi=T^{*}$ and thus also $(\sigma \tau) \psi=T^{*}$ in both cases. Hence $(\sigma \psi)_{\tau K^{*}} \subseteq(\sigma \tau) \psi=T^{*}$, which by Lemma 4.7 gives $\sigma \psi \subseteq \mathcal{S}^{\tau}$. Now considering all relevant factorizations of $\langle l, n-1\rangle$ and $\langle r, n\rangle$, we obtain the following results which we present in the form of a table.

\begin{tabular}{|c|c|c|c|c|c|}
\hline$i$ & $\sigma$ & $\tau$ & \multicolumn{1}{|c|}{$\psi$} & $\mathcal{I}$ & $\varphi$ \\
\hline $2,4, \ldots, n-2$ & $\langle l, i\rangle$ & $\langle l, n-1-i\rangle$ & $\langle l, i\rangle \psi \subseteq \mathcal{S}^{(l, n-1-i)}$ & $\mathcal{I}_{n-i}$ & $\langle l, i\rangle \varphi$ \\
$1,3, \ldots, n-3$ & $\langle l, i\rangle$ & $\langle r, n-1-i\rangle$ & $\langle l, i\rangle \psi \subseteq \mathcal{S}^{(r, n-1-i\rangle}$ & $\overline{\mathcal{I}}_{n-i}$ & $\langle l, i\rangle \varphi$ \\
$2,4, \ldots, n-2$ & $\langle r, i\rangle$ & $\langle r, n-i\rangle$ & $\langle r, i\rangle \psi \subseteq \mathcal{S}^{(l, n-i)}$ & $\mathcal{I}_{n+1-i}$ & $\langle r, i\rangle \varphi$ \\
$1,3, \ldots, n-1$ & $\langle r, i\rangle$ & $\langle l, n-i\rangle$ & $\langle r, i\rangle \psi \subseteq \mathcal{S}^{(r, n-i\rangle}$ & $\overline{\mathcal{I}_{n+1-i}}$ & $\langle r, i\rangle \varphi$ \\
\hline
\end{tabular}


The first column of the table gives the range for $i$, the second the choice of $\sigma$, the third the choice for $\tau$, the fourth uses condition (ii) above and Lemma 4.7, the fifth equals the bound in the fourth column by Lemma 4.2 (i)-(ii), the sixth the corresponding values of $\varphi$. That the fifth and the sixth columns are equal follows by an easy inductive process, see Figure 2.
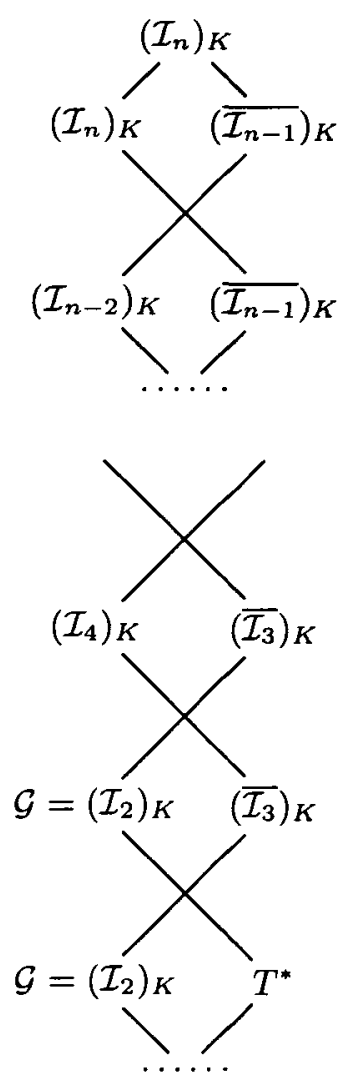

FIGURE 2. The ladder of $\mathcal{I}_{n}$ for $n$ even

We have thus proved that $\sigma \psi \subseteq \sigma \varphi$ for all $\sigma \in \Theta^{1}$ for which $\sigma \psi \in \mathcal{K}_{0}$; for the remaining $\sigma$ we have $\sigma \psi=\sigma \varphi$. Therefore $\psi \subseteq \varphi$ which by Theorem 3.1 implies that $\mathcal{V} \subseteq \mathcal{U}$.

We first define a relation $B$ by

$$
\mathcal{U} B \mathcal{V} \Longleftrightarrow \mathcal{U} \cap \mathcal{B}=\mathcal{V} \cap \mathcal{B}(\mathcal{U}, \mathcal{V} \in \mathcal{L}(\mathcal{C R}))
$$


We also define the correspondence $\mathcal{V} \rightarrow \mathcal{V}^{B}=(\mathcal{V} \cap \mathcal{B})^{B}(\mathcal{V} \in \mathcal{L}(\mathcal{C R}))$ as follows:

$$
(\mathcal{V} \cap \mathcal{B})^{B}=\mathcal{V} \quad \text { if } \quad \mathcal{V} \in\{\mathcal{G}, \mathcal{L G}, \mathcal{R G}, \mathcal{C S}, \mathcal{P}, \mathcal{X} \cap \mathcal{Y}, \mathcal{C R}\}
$$

where $\mathcal{P}$ is canonical, $\mathcal{P} \neq \mathcal{H}_{2}, \overline{\mathcal{H}_{2}}$, and

$$
\begin{aligned}
(\mathcal{X}, \mathcal{Y}) & \in\left\{\left(\mathcal{I}_{n}, \overline{\mathcal{H}_{n+1}}\right),\left(\mathcal{I}_{n}, \overline{\mathcal{I}_{n}}\right),\left(\mathcal{H}_{n+1}, \overline{\mathcal{I}_{n}}\right) \mid n \geq 2\right\} \\
\cup & \left\{\left(\mathcal{H}_{n}, \overline{\mathcal{I}_{n}}\right),\left(\mathcal{H}_{n}, \overline{\mathcal{H}_{n}}\right),\left(\mathcal{I}_{n}, \overline{\mathcal{H}_{n}}\right) \mid n>2\right\} .
\end{aligned}
$$

According to Figure $1, \mathcal{V}^{B}$ is defined for all $\mathcal{V} \in \mathcal{L}(\mathcal{C R})$ and is thus a function on $\mathcal{L}(\mathcal{C R})$ into itself.

The part of the ladder $L$ labelled by elements of $\mathbb{N}_{3}^{*}$ including its position in $L$ is the socle of $L$ to be denoted by $\operatorname{soc}(L)$. We are now ready for the principal result of the paper.

THEOREM 5.3. The mapping $\eta: \mathcal{V} \rightarrow \mathcal{V} \cap \mathcal{B}(\mathcal{V} \in \mathcal{L}(\mathcal{C R})$ ) is a complete retraction of $\mathcal{L}(\mathcal{C R})$ onto $\mathcal{L}(\mathcal{B})$, which induces $B$. Moreover, for any $\mathcal{U}, \mathcal{V} \in \mathcal{L}(\mathcal{C} R)$, we have

(i) $\mathcal{U} B \mathcal{V}$ if and only if $\operatorname{soc}\left(\chi_{\mathcal{U}}\right)=\operatorname{soc}\left(\chi_{\mathcal{V}}\right)$,

(ii) $\mathcal{V} B=\left[\mathcal{V} \cap \mathcal{B},(\mathcal{V} \cap \mathcal{B})^{B}\right]$

Proof. By [8], (and of origin [12, 14]), $\mathcal{B}$ is a neutral element of $\mathcal{L}(\mathcal{C R})$. This implies that the mapping $\eta$ is a homomorphism of $\mathcal{L}(\mathcal{C R})$ into $\mathcal{L}(\mathcal{B})$. Evidently, $\eta$ leaves the members of $\mathcal{L}(\mathcal{B})$ fixed. We have seen in Figure 1 that the lattice $\mathcal{L}(\mathcal{B})$ is of finite width. Hence the join of an arbitrary family $\mathcal{F}$ of band varieties equals the join of a finite subfamily of $\mathcal{F}$. It follows that $\eta$ is a complete $\vee$-homomorphism and is trivially a complete $n$-homomorphism. Clearly $\eta$ induces the relation $B$.

(i) From the discussion at the beginning of this section, we immediately see that any two varieties have the same intersection with $\mathcal{B}$ if and only if their ladders have the same socle.

(ii) Given $\mathcal{V} \in \mathcal{L}(\mathcal{C R})$, it is obvious that $\mathcal{V} \cap \mathcal{B}$ is the least variety having the same intersection with $\mathcal{B}$ as $\mathcal{V}$. For the upper end of the interval $\mathcal{V} B$, we proceed as follows.

We show first that for all $\mathcal{W} \in \mathcal{L}(\mathcal{B})$, we have $\mathcal{W}^{B} \cap \mathcal{B}=\mathcal{W}$. This obviously holds for $\mathcal{W} \in \mathcal{L}(\mathcal{R B})$. For a canonical variety $\mathcal{P}$ and $\mathcal{W}=\mathcal{P} \cap \mathcal{B}$, we get

$$
\mathcal{W}^{B} \cap \mathcal{B}=(\mathcal{P} \cap \mathcal{B})^{B} \cap \mathcal{B}=\mathcal{P} \cap \mathcal{B}=\mathcal{W}
$$

and for the remaining varieties $\mathcal{W}=\mathcal{X} \cap \mathcal{Y} \cap \mathcal{B}$,

$$
\mathcal{W}^{B} \cap \mathcal{B}=(\mathcal{X} \cap \mathcal{Y} \cap \mathcal{B})^{B} \cap \mathcal{B}=\mathcal{X} \cap \mathcal{Y} \cap \mathcal{B}=\mathcal{W}
$$

We still have to show the required maximality.

Let $\mathcal{W} \in \mathcal{L}(\mathcal{B})$ and $\mathcal{V} \in \mathcal{L}(\mathcal{C R})$ be such that $\mathcal{V} \cap \mathcal{B}=\mathcal{W}$. We show that $\mathcal{V} \subseteq \mathcal{W}^{B}$ by starting from the bottom of Figure 1. The required statement holds if $\mathcal{W} \in \mathcal{L}(\mathcal{R B})$. 
Lemma 5.2 takes care of the case $\mathcal{W}=\mathcal{P} \cap \mathcal{B}$ where $\mathcal{P}$ is a canonical variety. For the remaining varieties, let $\mathcal{X}$ and $\mathcal{Y}$ be canonical varieties and consider the band variety $\mathcal{W}=\mathcal{X} \cap \mathcal{Y} \cap \mathcal{B}$. We have seen above that $\mathcal{B}$ is a neutral element of $\mathcal{L}(\mathcal{C R})$. Hence

$$
[\mathcal{V} \vee(\mathcal{X} \cap \mathcal{B})] \cap \mathcal{B}=(\mathcal{V} \cap \mathcal{B}) \vee(\mathcal{X} \cap \mathcal{B})=\mathcal{X} \cap \mathcal{B}
$$

Since $\mathcal{X}$ is a canonical variety, by the above we obtain that $\mathcal{V} \vee(\mathcal{X} \cap \mathcal{B}) \subseteq \mathcal{X}$, and thus $\mathcal{V} \subseteq \mathcal{X}$. Analogously, we get $\mathcal{V} \subseteq \mathcal{Y}$ whence $\mathcal{V} \subseteq \mathcal{X} \cap \mathcal{Y}$, as required.

Therefore $\mathcal{W}^{B}$ has the requisite properties, which implies that for any $\mathcal{V} \in \mathcal{L}(\mathcal{C R})$, we have $\mathcal{V} B=\left[\mathcal{V} \cap \mathcal{B},(\mathcal{V} \cap \mathcal{B})^{B}\right]$, as asserted.

If, for $\mathcal{V} \in \mathcal{L}(\mathcal{B})$, we let $\mathcal{V}_{B}=\mathcal{V}$, we obtain the familiar form $\mathcal{V} B=\left[\mathcal{V}_{B}, \mathcal{V}^{B}\right]$. We now relate the ladders with the variety $\mathcal{V}^{B}$.

PROPOSITION 5.4. Let $\mathcal{V} \in[\mathcal{S}, \mathcal{B}]$ and $\mathcal{U}$ be the intersection of all canonical varieties containing $\mathcal{V}$. Then $\mathcal{V}^{B}=\mathcal{U}=\bigcap\left\{(\tau \chi \mathcal{V})^{K^{*} \bar{\tau}} \mid \tau \chi \mathcal{V} \in \mathbb{N}_{3}^{*}\right\}$.

PROOF. Let $\left\{\mathcal{U}_{\alpha} \mid \alpha \in A\right\}$ be the set of all canonical varieties containing $\mathcal{V}$. According to Figure $1, \mathcal{V}=\bigcap_{\alpha \in A}\left(\mathcal{U}_{\alpha} \cap \mathcal{B}\right)$ and thus, by the dual of Pastijn and Petrich [3, Lemma 4.10], we obtain

$$
\mathcal{V}^{B}=\left(\bigcap_{\alpha \in A}\left(\mathcal{U}_{\alpha} \cap \mathcal{B}\right)\right)^{B}=\bigcap_{\alpha \in A}\left(\mathcal{U}_{\alpha} \cap \mathcal{B}\right)^{B}=\bigcap_{\alpha \in A} \mathcal{U}_{\alpha}=\mathcal{U}
$$

This proves the first equality in the statement of the proposition. For the second, we proceed as follows.

If $\tau \chi_{\nu} \in \mathbb{N}_{3}^{*}$, then $\left(\tau \chi_{\nu}\right)^{K^{*}} \in \mathbb{N}_{3}$ and thus, by Lemma 4.2, we have that $\left(\tau \chi_{\nu}\right)^{K^{*} \bar{\tau}}$ is canonical. Hence

$$
\mathcal{U} \subseteq \bigcap\left\{\left(\tau \chi_{\mathcal{V}}\right)^{K^{*} \bar{\tau}} \mid \tau \chi_{\mathcal{V}} \in \mathbb{N}_{3}^{*}\right\}
$$

For the opposite inclusion, let $\mathcal{W}$ be a canonical variety such that $\mathcal{V} \subseteq \mathcal{W}$. By the cited reference, $\mathcal{W}=\mathcal{X}^{\tau}$ for some $\mathcal{X} \in \mathbb{N}_{3}$ and $\tau \in \Theta$. Lemma 3.3 (iv) implies that $\mathcal{V}_{\bar{\tau}} \subseteq\left(\mathcal{X}^{\tau}\right)_{\bar{\tau}}$ which, by [8], (of origin [10]) yields that $\mathcal{V}_{\bar{\tau}}$ is contained either in $\mathcal{X}_{T_{l}}$ or in $\mathcal{X}_{T_{r}}$. In either case, we get $\mathcal{V}_{\bar{\tau}} \in \mathbb{N}_{3}$ whence $\mathcal{V}_{\bar{\tau} K^{*}} \in \mathbb{N}_{3}^{*}$. Further, using the cited references, we get $(\bar{\tau} \chi \mathcal{V})^{K^{*} \tau}=\left(\mathcal{V}_{\bar{\tau} K^{*}}\right)^{K^{*} \tau} \subseteq\left(\left(\mathcal{X}^{\tau}\right)_{\bar{\tau} K^{*}}\right)^{K^{*} \tau} \subseteq \mathcal{X}^{\tau}=\mathcal{W}$. The opposite inclusion in (5.1) follows. This proves the second equality in the statement of the proposition.

If we include the varieties $\mathcal{G}, \mathcal{L G}, \mathcal{R G}, \mathcal{C S}$ and $\mathcal{C R}$ in the set of canonical varieties, then by Proposition 5.4, for any $\mathcal{V} \in \mathcal{L}(\mathcal{B}), \mathcal{V}^{B}$ is the intersection of all canonical varieties containing $\mathcal{V}$. 
COROLlary 5.5. The mapping $\mathcal{V} \rightarrow \mathcal{V}^{B}(\mathcal{V} \in \mathcal{L}(\mathcal{C R}))$ is a complete $\mathrm{n}$-endomorphism but is not a $\vee$-homomorphism of $\mathcal{L}(\mathcal{C R})$.

Proof. The first assertion follows easily from the first equality in Proposition 5.4 and the above remark. For the second,

$$
\mathcal{L Z}^{B} \vee \mathcal{R} \mathcal{Z}^{B}=\mathcal{L} \mathcal{G} \vee \mathcal{R G}=\mathcal{R} e \mathcal{G}, \quad(\mathcal{L} \vee \mathcal{R Z})^{B}=\mathcal{R} \mathcal{B}^{B}=\mathcal{C S}
$$

\section{Intersections with $\mathcal{B G}, \mathcal{O B G}$ and $\mathcal{B}$}

For any canonical variety $\mathcal{P}_{n}$ we provide here a single identity that serves as a basis of identities for $\mathcal{P}_{n} \cap \mathcal{B G}, \mathcal{P}_{n} \cap \mathcal{O B G}$ and $\mathcal{P}_{n} \cap \mathcal{B}$, respectively. These identities are variants of $G_{n}=P_{n}$ with suitable interspersing of $x_{i}^{0}$ instead of $x_{i}$ and $G_{n}^{0}$ and $P_{n}^{0}$, instead of $G_{n}$ and $P_{n}$, respectively. We also characterize the first two intersections.

We consider only $\mathcal{P}_{n}$; the statements for $\overline{\mathcal{P}_{n}}$ are dual. There is nothing to say about $\mathcal{H}_{2}$, while $\mathcal{I}_{2}$ requires a somewhat different treatment. Hence we start with $\mathcal{I}_{2}$ and then treat the remaining cases in a unified way.

PROPOSITION 6.1.

(i) $\mathcal{I}_{2} \cap \mathcal{B G}=\mathcal{I}_{2} \cap \mathcal{O B G}=\left[\left(x_{2} x_{1}\right)^{0}=x_{2}^{0} x_{1}^{0} x_{2}^{0}\right]=\mathcal{L} \mathcal{R B G}=\mathcal{L} \mathcal{R B} \vee \mathcal{G}$

(ii) $\mathcal{I}_{2} \cap \mathcal{B}=\left[x_{2} x_{1}=x_{2} x_{1} x_{2}\right]$.

ProOF. (i) The first equality follows from the well-known fact that $\mathcal{I}_{2} \subseteq \mathcal{O}$. The identities for $\mathcal{I}_{2}$ and $\mathcal{O B G}$ yield $\left(x_{2} x_{1}\right)^{0}=\left(x_{2} x_{1} x_{2}^{0}\right)^{0}=x_{2}^{0} x_{1}^{0} x_{2}^{0}$, proving that the second variety is contained in the third. The third, fourth and fifth equalities form part of Petrich and Reilly [7, Lemma V.5.2]. The last variety is obviously contained in the first.

(ii) This is well-known.

For the remaining cases, it is convenient to use the following notation:

(i) for a word $w$, possibly involving products, inversion and parentheses, we write $\hat{w}$ for the word obtained from $w$ by deleting all powers of words, positive, negative or zero, as well as all parentheses;

(ii) $G_{0}=P_{0}=\emptyset$, the empty word, for $P \in\{H, I\}$;

(iii) $G_{n}\left(x_{i}^{0}\right)$ stands for the word $G_{n}$, in which every variable $x_{i}$ is replaced by $x_{i}^{0}$.

THEOREM 6.2. For $\mathcal{P} \in\{\mathcal{H}, \mathcal{I}\}$ and $n>2$, the following equalities hold. Set $G_{1}=x_{1}$.

(i) $\mathcal{P}_{n} \cap \mathcal{B G}=\left[\left(x_{n}^{0} G_{n-2} x_{n-1}^{0}\right)^{0}=P_{n}^{0}\right]=\left[G_{n}^{0}=\left(P_{n}\left(x_{1}, \ldots, x_{n-2}, x_{n-1}^{0}, x_{n}^{0}\right)\right)^{0}\right]$ $=\left(\mathcal{P}_{n} \cap \mathcal{B}\right)$-band of groups. 
(ii) $\mathcal{P}_{n} \cap \mathcal{O B G}=\left[G_{n}\left(x_{i}^{0}\right)=P_{n}^{0}\right]=\left(\mathcal{P}_{n} \cap \mathcal{B}\right) \vee \mathcal{G}$.

(iii) $\mathcal{P}_{n} \cap \mathcal{B}=\left[G_{n}=\hat{P}_{n}\right]$.

PROOF. (i) Denote these four varieties by $\mathcal{U}, \mathcal{V}, \mathcal{W}$ and $\mathcal{X}$, respectively. $\mathcal{U} \subseteq \mathcal{V}$. First

$$
G_{n}=x_{n} \overline{G_{n-1}}=x_{n} \overline{x_{n-1} \overline{G_{n-2}}}=x_{n} G_{n-2} x_{n-1},
$$

which together with the identity of $\mathcal{B G}$ yields

$$
G_{n}^{0}=\left(x_{n} G_{n-2} x_{n-1}\right)^{0}=\left(x_{n}^{0} G_{n-2} x_{n-1}^{0}\right)^{0} .
$$

It remains to observe that $G_{n}=P_{n}$ implies $G_{n}^{0}=P_{n}^{0}$.

$\mathcal{V} \subseteq \mathcal{W}$. We consider first the case $n=3$. The substitution $x_{1} \rightarrow x_{2}^{0}$ in the identity of $\mathcal{V}$ yields the identity of $B \mathcal{G}$. The two together immediately imply the identity of $\mathcal{W}$. Hence assume that $n>3$. Then

$$
\begin{aligned}
P_{n} & =G_{n}\left(x_{n} \overline{P_{n-1}}\right)^{0}=G_{n}\left(x_{n}\left(\overline{G_{n-1}\left(x_{n-1} \overline{P_{n-2}}\right)^{0}}\right)^{0}\right. \\
& =G_{n}\left(x_{n}\left(P_{n-2} x_{n-1}\right)^{0} \overline{G_{n-1}}\right)^{0} \\
& =x_{n} G_{n-2} x_{n-1}\left(x_{n}\left(P_{n-2} x_{n-1}\right)^{0} G_{n-2} x_{n-1}\right)^{0} .
\end{aligned}
$$

The substitution $x_{i} \rightarrow x_{n-1}$ for $i=1,2, \ldots, n-2$ in the identity given by (6.2) yields

$$
\left(x_{n}^{0} x_{n-1}^{0}\right)^{0}=\left(x_{n} x_{n-1}\left(x_{n} x_{n-1}^{0} x_{n-1}\right)^{0}\right)^{0}=\left(x_{n} x_{n-1}\right)^{0},
$$

the identity of $\mathcal{B G}$. Now using (6.1) and (6.2), we obtain

$$
\begin{aligned}
G_{n}^{0} & =\left(x_{n} G_{n-2} x_{n-1}\right)^{0}=\left(x_{n}^{0} G_{n-2} x_{n-1}^{0}\right)^{0}=P_{n}^{0} \\
& =\left(P_{n}\left(x_{1}, \ldots, x_{n-2}, x_{n-1}^{0}, x_{n}^{0}\right)\right)^{0} .
\end{aligned}
$$

$\mathcal{W} \subseteq \mathcal{X}$. The same substitution as above in the identity given by (6.1) and (6.2) yields

$$
\left(x_{n} x_{n-1}\right)^{0}=\left(x_{n}^{0} x_{n-1}^{0}\left(x_{n}^{0} x_{n-1}^{0} x_{n-1}^{0}\right)^{0}\right)^{0}=\left(x_{n}^{0} x_{n-1}^{0}\right)^{0},
$$

the identity of $\mathcal{B G}$. Hence if $S \in \mathcal{W}$, then $S \in \mathcal{B G}$ and $S / \mathcal{H} \in \mathcal{P}_{n} \cap \mathcal{B}$ and thus $S \in \mathcal{X}$.

$\mathcal{X} \subseteq \mathcal{U}$. This case follows from Lemma 5.2.

(ii) Denote these three varieties by $\mathcal{U}, \mathcal{V}$ and $\mathcal{W}$, respectively. In the case $n=3$, the identity for $\mathcal{O B G}$ implies that $\mathcal{U}=\mathcal{V}=\mathcal{W}$ using similar arguments as those above. So assume that $n>3$. 
$\mathcal{U} \subseteq \mathcal{V}$. This is a direct consequence of $\mathcal{O B G}=\left[a^{0} x^{0}=(a x)^{0}\right]$.

$\mathcal{V} \subseteq \mathcal{W}$. The same substitution as above now yields $x_{n}^{0} x_{n-1}^{0}=\left(x_{n} x_{n-1}\right)^{0}$, see (6.3), giving the identity of $\mathcal{O B G}$.

By Petrich [5], we obtain $\mathcal{V}=(\mathcal{V} \cap \mathcal{B}) \vee(\mathcal{V} \cap \mathcal{G})=\left(\mathcal{P}_{n} \cap \mathcal{B}\right) \vee \mathcal{G}=\mathcal{W}$

$\mathcal{W} \subseteq \mathcal{U}$. This is obvious.

(iii) Denote these two varieties by $\mathcal{U}$ and $\mathcal{V}$, respectively.

$\mathcal{U} \subseteq \mathcal{V}$ This is trivial.

$\mathcal{V} \subseteq \mathcal{U}$. The substitution $x_{i} \rightarrow x_{n}^{0}$ for $i=1,2, \ldots, n-1$ by (6.1) and (6.2) yields $x_{n}=x_{n}^{2}$. The identity now gives $G_{n}=P_{n}$ also.

\section{References}

[1] J. A. Gerhard and M. Petrich, 'Certain characterizations of varieties of bands', Proc. Edinburgh Math. Soc. (2) 31 (1988), 301-319.

[2] __ 'Varieties of bands revisited', Proc. London Math. Soc. (3) 58 (1989), 323-350.

[3] F. Pastijn and M. Petrich, 'Congruences on regular semigroups', Trans. Amer. Math. Soc. 295 (1986), 607-633.

[4] F. J. Pastijn, 'The lattice of completely regular semigroup varieties', J. Aust., Math. Soc. 49 (1990), $24-42$.

[5] M. Petrich, 'Varieties of orthodox bands of groups', Pacific J. Math. 58 (1975), 209-217.

[6] M. Petrich and N. R. Reilly, 'Operators related to $E$-disjunctive and fundamental completely regular semigroups', J. Algebra 134 (1990), 1-27.

[7] - Completely regular semigroups, Vol. I, Canadian Math. Soc. Series of Monographs and Advanced Texts 23 (Wiley, New York, 1999).

[8] _ Completely regular semigroups, Vol. II (in preparation).

[9] L. Polák, 'On varieties of completely regular semigroups I', Semigroup Forum 32 (1985), 97-123.

[10] — ' 'On varieties of completely regular semigroups II', Semigroup Forum 36 (1987), 253-284.

[11] V. V. Rasin, 'Varieties of orthodox Clifford semigroups', Izv. Vyssh. Uchebn. Zaved. Matem. 11 (1982), 82-85 (Russian).

[12] N. R. Reilly, 'Completely regular semigroups', in: Lattices, Semigroups and Universal Algebra (ed. J. Almeida) (Plenum Press, New York, 1990) pp. 225-242.

[13] N. R. Reilly and S. Zhang, 'Decomposition of the lattice of pseudovarieties of finite semigroups induced by bands', Algebra Universalis 44 (2000), 217-239.

[14] P. G. Trotter, 'Subdirect decomposition of the lattice of varieties of completely regular semigroups', Bull. Austral. Math. Soc. 39 (1989), 343-351.

[15] P. G. Trotter and P. Weil, 'The lattice of pseudovarieties of idempotent semigroups and a non-regular analogue', Algebra Universalis 37 (1997), 491-526.

\section{Bol, Brax}

\section{Croatia}

